\title{
Cerebral Activity during the Anesthesia-Like State Induced by Mesopontine Microinjection of Pentobarbital
}

\author{
Ruth Abulafia, Vladimir Zalkind, and Marshall Devor \\ Department of Cell and Animal Biology, Institute of Life Sciences and Center for Research on Pain, Hebrew University of Jerusalem, Jerusalem 91904, Israel
}

\begin{abstract}
Microinjection of pentobarbital into a restricted region of rat brainstem, the mesopontine tegmental anesthesia area (MPTA), induces a reversible anesthesia-like state characterized by loss of the righting reflex, atonia, antinociception, and loss of consciousness as assessed by electroencephalogram synchronization. We examined cerebral activity during this state using FOS expression as a marker. Animals were anesthetized for $50 \mathrm{~min}$ with a series of intracerebral microinjections of pentobarbital or with systemic pentobarbital and intracerebral microinjections of vehicle. FOS expression was compared with that in awake animals microinjected with vehicle. Neural activity was suppressed throughout the cortex whether anesthesia was induced by systemic or MPTA routes. Changes were less consistent subcortically. In the zona incerta and the nucleus raphe pallidus, expression was strongly suppressed during systemic anesthesia, but only mildly during MPTA-induced anesthesia. Dissociation was seen in the tuberomammillary nucleus where suppression occurred during systemic-induced anesthesia only, and in the lateral habenular nucleus where activity was markedly increased during systemicinduced anesthesia but not following intracerebral microinjection. Several subcortical nuclei previously associated with cerebral arousal were not affected. In the MPTA itself FOS expression was suppressed during systemic anesthesia. Differences in the pattern of brain activity in the two modes of anesthesia are consistent with the possibility that anesthetic endpoints might be achieved by alternative mechanisms: direct drug action for systemic anesthesia or via ascending pathways for MPTA-induced anesthesia. However, it is also possible that systemically administered agents induce anesthesia, at least in part, by a primary action in the MPTA with cortical inhibition occurring secondarily.
\end{abstract}

\section{Introduction}

General anesthetic agents profoundly alter behavioral state. Locomotion ceases, there is a loss of postural support and the righting reflex, muscle tone is reduced, and responses to noxious stimuli are obtunded. In addition, memory formation ceases and there is loss of consciousness, associated with synchronization of cortical electroencephalogram. This behavioral collapse is generally presumed to reflect the distribution of the anesthetic molecules in the systemic circulation, with functional suppression reflecting direct receptor binding to neurons in the cortex and elsewhere in the CNS (Franks and Lieb, 1994; Antkowiak, 1999; Hentschke et al., 2005). Concussion, syncope (fainting), and other "anesthesia-like states" that share the key behavioral characteristics of anesthesia are likewise presumed to reflect distributed suppression, in these cases following metabolic insufficiency due to diffuse neuronal injury, hypoxia, hypotension, hypoglycemia, etc. (Ommaya and Gennarelli, 1974; Plum and Posner, 1980; Surtees and Leonard, 1989).

A competing hypothesis holds that as in sleep-wake transi-

Received March 29, 2008; revised April 7, 2009; accepted April 22, 2009.

This work was supported by grants from the Israel Science Foundation and the Hebrew University Center for Research on Pain. We thank Moran Horesh, Inna Sukhotinsky, Ron Amir, Israel Nelken, Shahar Barbash, Shay Leventhal, Adi Tapiero, and Yelena Fishman for advice and technical assistance and llan Hammel for advice on counting methodology.

Correspondence should be addressed to Prof. Marshall Devor, Department of Cell and Animal Biology, Institute of Life Sciences and Center for Research on Pain, Hebrew University of Jerusalem, Jerusalem 91904, Israel. E-mail: marshlu@vms.huji.ac.il.

DOI:10.1523/JNEUROSCI.1357-08.2009

Copyright $\odot 2009$ Society for Neuroscience $\quad$ 0270-6474/09/297053-12\$15.00/0 tions, behavioral collapse in anesthesia and the anesthesia-like states is related to activity in dedicated brainstem and diencephalic structures, some associated with the reticular formation (Magni et al., 1959; Hayes et al., 1984; Katayama et al., 1988; Alkire et al., 2000; Nelson et al., 2002; Tung and Mendelson, 2004; Lydic and Baghdoyan, 2005; Morales et al., 2006; Franks, 2008; Zecharia et al., 2009). According to this view, global metabolic suppression in the brain is secondary to network activity transmitted by specific ascending pathways. Widespread neural suppression might not even be essential for behavioral collapse. For example, the anesthetic ketamine did not suppress FOS-IR except at high concentrations (Lu et al., 2008), and most anesthetics largely spare autonomic regulation and much signal processing in primary sensory cortices (Hubel and Wiesel, 1959; Plourde et al., 2006).

In accord with the second hypothesis, we have reported that an anesthesia-like state can be induced without an active agent being broadly distributed in the brain. This was accomplished by microinjecting pentobarbital or other $\mathrm{GABA}_{\mathrm{A}}$-receptor $\left(\mathrm{GABA}_{\mathrm{A}}-\mathrm{R}\right)$ active anesthetics into a focal brainstem locus, the mesopontine tegmental anesthesia area [MPTA (Devor and Zalkind, 2001; Voss et al., 2005; Sukhotinsky et al., 2007)]. The rapidity of onset and the minute drug volume and dose that are adequate preclude global redistribution of the drug as a possible mechanism. The question then arises as to whether the anesthesia-like state induced by MPTA microinjection leads to a pattern of change in cerebral activity different from that induced by systemic anesthesia.

In the present study, we used FOS expression as a means of monitoring neural activity during the wake state, systemic anes- 
thesia, and the anesthesia-like state induced by pentobarbital microinjection into the MPTA. During normal wakefulness, FOS, the protein product of $c$-fos expression, is present at relatively high levels in most cortical areas (Pompeiano et al., 1994; Cirelli and Tononi, 2000). Systemic pentobarbital causes widespread suppression of cortical FOS (Takayama et al., 1994; Lu et al., 2008). We have now evaluated cortical and subcortical changes in FOS expression following intracerebral microinjection in the MPTA.

\section{Materials and Methods}

\section{Animals and surgery}

Experiments were performed using adult male rats of the Wistar-derived Sabra strain (275$525 \mathrm{~g}$ ), with the approval of the Institutional Animal Care and Use Committee of the Hebrew University of Jerusalem and following the ethical guidelines of the International Association for the Study of Pain (Zimmermann, 1983). Animals were deeply anesthetized with ketamine and xylazine $(85 \mathrm{mg} / \mathrm{kg}$ and $15 \mathrm{mg} / \mathrm{kg}$, i.p., respectively) and secured in a stereotaxic frame with the skull level between bregma and lambda. They were then implanted with a bilaterally symmetrical 23 ga stainless-steel guidecannula assembly [outer diameter (o.d.) $=0.64$ $\mathrm{mm}$ ] aimed at the MPTA. The cannulae, which were plugged internally with 30 ga stainlesssteel stylets, were anchored to the skull with stainless-steel screws and dental acrylic. Implantation coordinates were $8 \mathrm{~mm}$ posterior to bregma and $1.25 \mathrm{~mm}$ lateral to the midline suture, with the cannula tip inserted $\sim 4.0-4.5$ $\mathrm{mm}$ beneath the dura. Animals received a prophylactic dose of penicillin $(50 \mathrm{kU} / \mathrm{kg}$, i.m.) and were returned to their home cage, one animal to a cage. Recovery was uneventful. Both before and after surgery rats received routine veterinary care. Food pellets and water were available ad libitum (Koffolk, product \#19520), and the day:night cycle was $12 \mathrm{~h}: 12 \mathrm{~h}$ with lights on at 7:00 A.M.

\section{Intracerebral microinjection}

After a postoperative recovery time of at least

$7 \mathrm{~d}$, we attempted to evoke an anesthesia-like state by microinjecting pentobarbital into the MPTA. The animal was gently restrained in a soft cloth, the stylet was removed, and pentobarbital, mixed fresh from powder (Danisco Ingredients, $100 \mu \mathrm{g}$ in $0.5 \mu \mathrm{l}$ of vehicle containing $10 \%$ ethanol, $20 \%$ propylene glycol, $70 \% \mathrm{H}_{2} \mathrm{O}, \mathrm{pH} 10.3$ ) was microinjected on both sides using a sterilized 30 ga stainless-steel injection cannula (o.d. = $0.31 \mathrm{~mm}$ ) attached with polyethylene tubing (PE10) to a $10 \mu$ l Hamilton microsyringe. The stylets were then replaced. Solution was injected at $\sim 0.5 \mu \mathrm{l} / 30 \mathrm{~s}$ and the injection cannula was left in place for another $30 \mathrm{~s}$ before withdrawal. The time from the beginning of the first microinjection until the end of the procedure was $\sim 2-3$ min. Immediately afterward, animals were released into a transparent plastic observation cage for behavioral assessment.

For each animal, it was necessary to find a microinjection depth that would induce an anesthesia-like state. The first microinjection trial was made at a depth of 6.0 or $6.5 \mathrm{~mm}$ below the dura. If a criterion anesthesialike state was not obtained on this attempt, an additional trial was made after insertion of the injection cannula an additional $0.5 \mathrm{~mm}$ in depth. One or two such screening trials were usually enough, but if necessary a third was made $0.5 \mathrm{~mm}$ deeper still. An interval of 3-7 d was allowed between successive trials. Animals in which a criterion anesthesia-like state was not achieved were used in one of the comparison groups [intraperitoneal pentobarbital (IP), awake, or microinjection (-)]. All three of the main experimental groups (MPTA, IP, awake) had a similar number of screening trials (1.6, 1.5 , and 1.5 on average, respectively). Animals of the microinjection (-) group had an average of 2.3 screening trials. The locus of all microinjections was determined histologically at the end of the study (Fig. 1A).

The defect following multiple cannula insertions and pentobarbital microinjections was $\sim 1.1 \mathrm{~mm}$ in diameter, and this was surrounded by a shell of FOS-immunoreactive cells $\sim 0.3 \mathrm{~mm}$ across for a total diameter of $\sim 1.7 \mathrm{~mm}$ (volume $\sim 2.6 \mu \mathrm{l}$ ) (Fig. $1 B$ ).

\section{Assessment}

The behavior of the animals was recorded on videotape from before the microinjection until the end of the observation period. The anesthesialike state was quantified by four standard behavioral scales, two motor [posture and loss of righting reflex (LORR)] and two sensory (response to a brief calibrated hindlimb pinch and tail pinch). Scoring on each scale 
ranged from 0 , indicating the normal behavior of a fully alert rat, to 4 , indicating maximal motor and sensory depression, as described in detail by Devor and Zalkind (2001). The maximum possible score was 16 , or 8 when the sensory and motor scales were considered separately. Our criterion for an anesthesia-like state in the present study was a combined score of $\geq 11$ with the condition that this includes loss of the righting reflex (score of $\geq 3$ on the LORR scale).

We acknowledge that the assessment protocol, and particularly paw and tail pinch stimuli, might have affected FOS expression, although the latter stimuli were identical across the experimental groups.

\section{Experimental protocol}

The aim of the study was to compare cerebral activity in awake rats to rats anesthetized for 50 min with pentobarbital injected systemically or microinjected into the MPTA. The state of alertness was assessed as described above approximately every 5 min throughout the observation period (see Fig. 2). The animals were then killed by transcardial perfusion. Rats were divided into three main groups (MPTA, IP, and awake) and three supplemental groups.

MPTA group (five rats). A criterion anesthesia-like state was induced by pentobarbital microinjected into the MPTA on two sides through previously implanted cannulae as described above. Since rats often began to awaken $\sim 15-20 \mathrm{~min}$ after a single microinjection, to ensure at least 50 min of anesthesia a series of three intracerebral microinjections was carried out, each microinjection separated by $\sim 15 \mathrm{~min}$ (see Fig. $2 \mathrm{~A}$, downward-pointing arrows). To match the IP group, $1.0 \mathrm{ml} / \mathrm{kg}$ saline was injected intraperitoneally $\sim 10 \mathrm{~min}$ before the beginning of the 50 min observation period (see Fig. $2 A$, upward-pointing arrow). In both the IP group and the MPTA group, we aimed to maintain the anesthesia score $\geq 11$ throughout the 50 min observation period.

IP group (four rats). Animals were anesthetized to the criterion anesthetic plane by intraperitoneal injection of pentobarbital $(60-70 \mathrm{mg} / \mathrm{kg}$, Pental Veterinary; CTS Chemical Industries, $200 \mathrm{mg} / \mathrm{ml}$, diluted to 60 $\mathrm{mg} / \mathrm{ml}$ with saline). To match the MPTA group, three $0.5 \mu \mathrm{l}$ volumes of the vehicle used for dissolving pentobarbital powder (above) were microinjected intracerebrally at intervals of $\sim 15 \mathrm{~min}$. The timing of the systemic and intracerebral injections in these animals is shown by arrows in Figure $2 B$.

Awake group (four rats). These animals remained awake during the 50 min observation period. To ensure an alert state, the animals were monitored continuously. They were permitted to stand or sit, but if they closed their eyes they were aroused by a gentle prodding vigorous enough to cause them to stand and walk a few paces. To match the other two groups, these animals received three intracerebral microinjections of 0.5 $\mu \mathrm{l}$ of vehicle as above, and in addition $1.0 \mathrm{ml} / \mathrm{kg}$ saline (i.p.) $\sim 10 \mathrm{~min}$ before the beginning of the 50 min observation period (see Fig. 2C).

Supplemental groups. Data were also obtained from an additional three comparison groups. Two groups of animals without cannula implants received either an anesthetic dose of pentobarbital intraperitoneally (three rats), or the same volume of saline (five rats). The latter were kept awake. The last group (three rats) consisted of animals with cannula implants but in which, despite the localization of the cannula tips in the MPTA, microinjection of pentobarbital failed to induce a criterion anesthesia-like state. We refer to these animals as the microinjection $(-)$ group. These three animals were like those in the MPTA group in that they received three intracerebral microinjections of pentobarbital and $1.0 \mathrm{ml} / \mathrm{kg}$ saline (i.p.). However, their anesthesia score remained $<11$ throughout the observation period (see Fig. 2D).

\section{Histology}

Perfusion and tissue sectioning. Particularly in the awake and MPTA groups, it was essential to fix the brain rapidly with a minimum period of systemic-induced anesthesia. To this end, immediately after the $50 \mathrm{~min}$ observation period all animals received a heavy overdose of pentobarbital. As soon as they were deeply anesthetized (score $=16$ ) they were perfused transcardially with $0.9 \%$ saline followed by $4 \%$ formaldehyde in $0.1 \mathrm{~m}$ phosphate buffer (PB), pH 7.3. Fixative reached the brain no $>13$ $\min$ (nor $<8 \mathrm{~min}$ ) after injection of the terminal pentobarbital dose. The brain was dissected out immediately and postfixed for at least $3 \mathrm{~h}$ in the same fixative. It was then transferred to cryoprotectant, $20 \%$ sucrose in PBS containing $0.02 \%$ sodium azide (PBS-azide) at $4^{\circ} \mathrm{C}$. At least $48 \mathrm{~h}$ later, tissue was cut on a freezing microtome into five series of $50-\mu \mathrm{m}$ thick coronal sections ( $250 \mu \mathrm{m}$ separation), and stored in PBS-azide at $4^{\circ} \mathrm{C}$.

Immunolabeling. Sections were rinsed in PBS, incubated in $0.12 \%$ $\mathrm{H}_{2} \mathrm{O}_{2}$ for $1 \mathrm{~h}$ to inactivate endogenous peroxidases, rinsed again, and then incubated in polyclonal rabbit anti-FOS (Oncogene Research Products, Ab-5, immunizing antigen residues $4-17$ of human FOS protein) $1: 100,000$ in PBS containing $0.25 \%$ Triton X-100 and $0.2 \%$ sodium azide (TBS-azide) for $\sim 20 \mathrm{~h}$ at room temperature. Rinsed sections were then incubated for $60 \mathrm{~min}$ in a secondary antibody, biotinylated goat antirabbit IgG (Jackson ImmunoResearch, 1:1000 in TBS), rinsed, and incubated for an additional $60 \mathrm{~min}$ in ABC reagent in PBS $(1 \mu \mathrm{l} / \mathrm{ml}$, Vectastain Elite ABC Kit, Vector Laboratories). Sections were then rinsed again and reacted in a PBS solution containing $0.05 \%$ 3,3diaminobenzidine tetrahydrochloride (DAB, Sigma), $0.01 \% \mathrm{H}_{2} \mathrm{O}_{2}$, $0.01 \% \mathrm{Ni}\left(\mathrm{NH}_{4}\right) \mathrm{SO}_{4}$, and $0.005 \% \mathrm{CoCl}_{2}$ for 15-40 min with visual monitoring, rinsed, mounted on gel-covered slides, and lightly counterstained with cresyl violet. Finally, they were rinsed in $\mathrm{H}_{2} \mathrm{O}$, dehydrated in ascending alcohols, cleared with xylene, and coverslipped using Entellan (Merck). Selectivity of the anti-FOS antibody is known from labeling restricted to cell nuclei in appropriate cell groups, and the absence of labeling in brain sections from $c$-fos knock-out mice.

For the IP, MPTA, and awake groups, a series of brainstem sections was double immunolabeled. After visualization of FOS-IR as described above, sections were incubated for $24 \mathrm{~h}$ in polyclonal rabbit anti-tyrosine hydroxylase antibody (TH, Millipore Bioscience Research Reagents, product \#AB152, lot \#20020676, 1:6000 in TBS-azide) followed by biotinylated goat anti-rabbit secondary antibody (Jackson ImmunoResearch Laboratories, 1:1000, $60 \mathrm{~min}$ in TBS). Sections were then reacted with the ABC-DAB procedure described above, but without $\mathrm{Ni}$ and $\mathrm{Co}$. This yielded a brown cytoplasmic reaction product, easily distinguished from the black nuclear marking of FOS-IR. The rest of the procedure, including cresyl violet counterstaining, was as described above. Specificity of the anti-TH antibody was characterized by biochemical means (Haycock, 1987), and it was confirmed in our hands by showing labeling limited to appropriate neuronal populations. For both FOS and TH protocols, immunolabeling was eliminated when the primary antibody was omitted. This assured the specificity of the secondary antibodies used.

\section{Data analysis}

FOS and TH immunolabeling was assessed from bright-field images captured on a Zeiss Universal microscope equipped with a motorized stage and a DEI-750CE CCD camera (Optronics) using the Neurolucida system (MicroBrightField), or a Zeiss Standard microscope with a Nikon D100 camera. Neurolucida and ImagePro Plus (Media Cybernetics) were used for image analysis and quantification. Evaluation was done blind from coded images.

Cell counting. For relatively large regions of interest (ROIs) such as the MPTA, and cortical fields, an appropriately sized counting frame, usually rectangular, was placed over the photomicrograph using predefined landmarks. This assured that a corresponding region was counted from animal to animal. For the primary somatomotor cortex (SM1 cx), we counted all FOS-immunoreactive neurons in a 300- $\mu$ m-wide rectangular slab of cortex stretching from the pia to the subcortical white matter $(\sim 1525 \mu \mathrm{m})$. This was divided into 10 equal rectangles. The upper rectangle represented the molecular layer. The next three represented the supragranular layers, the next two represented the cortical granular layer, and the deepest four represented the subgranular layers. For the prefrontal cortex $(\mathrm{PF} \mathrm{cx})$, a counting frame of $320 \times 480 \mu \mathrm{m}$ was placed over the supragranular layers. For the piriform cortex (Pir cx), the same counting frame was placed over the pyramidal cell layer at the level of the anterior commissure. The counting frame for the dentate gyrus (DG $\mathrm{cx}$ ) was $500 \times 755 \mu \mathrm{m}$. For small nuclei with well defined borders, i.e., the tuberomammillary nucleus (TMN) and the locus ceruleus (LC), the nucleus was outlined with a freehand tool, and the area of the ROI was computed. For nuclei with less distinct borders, a standardized counting frame sized to fit the nucleus was used (see Figs. 3, 5; supplemental Fig. 1, 
Table 1. Regions of interest and abbreviations used

\begin{tabular}{ll}
\hline ROI & Abbreviations \\
\hline A5-noradrenergic cell group & A5 \\
Dentate gyrus & DG cx \\
Dorsal raphe nucleus & DRN \\
Intralaminar thalamic nuclei & ITh \\
Lateral habenular nucleus & LHb \\
Locus ceruleus & LC \\
Mesopontine tegmental anesthesia area & MPTA \\
Midline thalamic nuclei & MTh \\
Nucleus raphe pallidus & Rpa \\
Gigantocellular reticular nucleus alpha & GiA \\
Piriform cortex & Pir cx \\
Prefrontal cortex & PF cx \\
Primary somatomotor cortex & SM1 cx \\
Tuberomammillary nucleus & TMN \\
Ventrolateral preoptic nucleus & VLPO \\
Zona incerta & Zl \\
\hline
\end{tabular}

available at www.jneurosci.org as supplemental material). These ROIs included the thalamic midline and intralaminar nuclear groups (MTh, ITh, see figure legend for dimensions), the zona incerta (ZI, $320 \times 480$ $\mu \mathrm{m})$, and component nuclei of the ventral medial medulla [nucleus raphe pallidus (Rpa), $200 \times 500 \mu \mathrm{m}$; part of the gigantocellular reticular nucleus $\alpha(\mathrm{GiA}) ; 400 \times 400 \mu \mathrm{m}]$. A counting frame $1000 \times 1600 \mu \mathrm{m}$ was used for the MPTA (see Fig. 8). For the A5 noradrenergic cell group, the percentage of FOS-immunoreactive nuclei among $\mathrm{TH}$-immunoreactive cell bodies was calculated for the entire nucleus.

We counted all immunopositive nuclei that fell within the boundaries of the counting frame in which at least half of the cell nucleus was present in the section. We then divided the resulting cell counts by the area counted to yield labeling density, expressed in units of immunolabeled nuclei per $100 \times 100 \mu \mathrm{m}$ area $\left[(100 \mu \mathrm{m})^{2}\right]$. No correction for split nuclei was applied on the grounds that the counting particle (nucleus, diameter $\sim 9 \mu \mathrm{m}$ ) was much smaller than the section thickness $(50 \mu \mathrm{m})$, and that nuclei tend to be pushed into one section or the other rather than being split (Koningsmark, 1970). Moreover, the key results were comparisons of density estimates from identical brain locations in different groups. Such comparisons are not expected to be affected by split-object correction factors. In sections double immunolabeled for FOS and TH, we determined for each TH-immunopositive neuron (brown cytoplasm) whether the nucleus was or was not FOS immunopositive (black nucleus). Brain areas quantified, and abbreviations used, are given in Table 1.

Statistical analysis. Anesthesia scores were analyzed using one-way ANOVA with post hoc Bonferroni $t$ test for multiple pairwise comparisons. Mean anesthesia score was calculated for each rat by integrating all assessments over the 50 min observation period. These values were then averaged over all rats in each experimental group. Scores for motor and sensory scales were sometimes used independently. Counts of FOS immunolabeling [nuclei per $(100 \mu \mathrm{m})^{2}$ ] for every area analyzed in the three main groups (MPTA, IP, awake) were tested for normality and equal variance. When data passed these tests we applied one-way ANOVA and post hoc the Bonferroni $t$ test. Data that failed normality and equal variance tests were analyzed using the nonparametric Kruskal-Wallis test and Dunn's method for multiple pairwise comparison post hoc (SigmaStat version 3.1, Systat Software). Comparisons between the MPTA and the microinjection ( - ) group were made using paired Student's $t$ tests or the Mann-Whitney $U$ test. Additional comparisons were made using the Fisher exact probabilities test, and Pearson correlation coefficient as indicated in the Results. Partial correlation coefficient were calculated using MATLAB (version 7, The MathWorks). Mean values are given \pm the SEM. We used a two-tailed significance criterion of $p=0.05$.

\section{Results}

Anesthesia and the anesthesia-like state

Anesthesia scores of animals in the three main experimental groups are shown in Figure $2 A-C$. A single systemic injection of pentobarbital in the IP group sustained a general anesthetic state throughout the $50 \mathrm{~min}$ observation period. The time from injection to reaching a criterion level of anesthesia (score $\geq 11$ ) was 5-10 $\mathrm{min}$, and the average anesthesia score during the $50 \mathrm{~min}$ observation period that followed was $15.1 \pm 0.6$ (Fig. 2 B). Maintaining a criterion anesthesia-like state for $50 \mathrm{~min}$ by intracerebral microinjection of pentobarbital (the MPTA group) was accomplished by repeated microinjections (Fig. $2 \mathrm{~A}$, downwardpointing arrows). The time from the first microinjection to achieving an anesthesia score $\geq 11$ varied, but averaged $\sim 3$ min (latency measured from the completion of the bilateral microinjections). The average anesthesia score during the 50 min observation period that followed was $12.4 \pm 0.3$, slightly less than the IP group $(p<0.05)$. In one rat, the score fell below 11 (to 8 ) at one assessment time point. The third microinjection was brought forward and delivered at that time, and the anesthesia score rapidly returned to 13 (Fig. $2 \mathrm{~A}$, open squares). In the awake group, there was no loss of alertness, with anesthesia scores averaging $1.3 \pm 0.6$ for the entire observation period (Fig. 2C). Scores were significantly higher in the IP and the MPTA groups than in the awake group $(p<0.001)$. Finally, in three rats pentobarbital targeted to the MPTA failed to induce a criterion anesthesia-like state [Fig. $2 D$, microinjection (-) group]. One rat was sedated with only partial LORR, and two remained fully awake. Anesthesia score averaged $2.6 \pm 2.6$, and was significantly different from the MPTA group $(p<0.01)$.

\section{Verification of microinjection sites}

Microinjection sites were identified as the most ventral point along the center of the trajectory of the injection cannula identified in the series of histological sections. They were plotted by projecting the section on the nearest standard brainstem outline [7.3 and $8.0 \mathrm{~mm}$ caudal to bregma by the brain atlas of Paxinos and Watson (1998)]. We verified that the locations plotted also matched the distance from the inner surface of the skull to the tip of the inserted injection cannula. The scatter of microinjection sites is given in Figure 1. All fell within the region of the MPTA (Devor and Zalkind, 2001), including those in the microinjection $(-)$ group.

\section{FOS expression in the cerebral cortex}

Awake rats showed high levels of FOS immunoreactivity in the cerebral cortex. This amounted to $\sim 20 \%$ of all neurons, assessed in two sampling frames counted in each of two cortical regions, SM1 cx and PF cx. This labeling density estimate was obtained by dividing the number of neurons with FOS-immunoreactive nuclei by the number labeled with cresyl violet. Compared with awake animals there was a substantial reduction in the density of FOS-immunoreactive neurons throughout the cerebral cortex in animals anesthetized by systemic drug administration as well as in animals in which an anesthesia-like state was induced by pentobarbital microinjection in the MPTA (Fig. 3A). Changes from the awake state were quantified in four ROIs: SM1 cx, PF cx, piriform cortex [Pir cx (pyramidal layer)], and DG cx. In the PF $\mathrm{cx}$ the decline was statistically significant during both modes of anesthesia $(p<0.001)$ (Fig. $4 A$ ). In the SM1 cx, the decline was significant in the IP group but not in the MPTA group. In the latter, FOS-IR density was midway between that of the IP and the awake groups. FOS immunolabeling also decreased in Pir cx and DG $\mathrm{cx}$ (Fig. 3B), but in contrast to SM1 cx, the change was statistically significant in the MPTA group but not in the IP group (Fig. $4 B)$. The density of FOS immunolabeling in the MPTA group did 

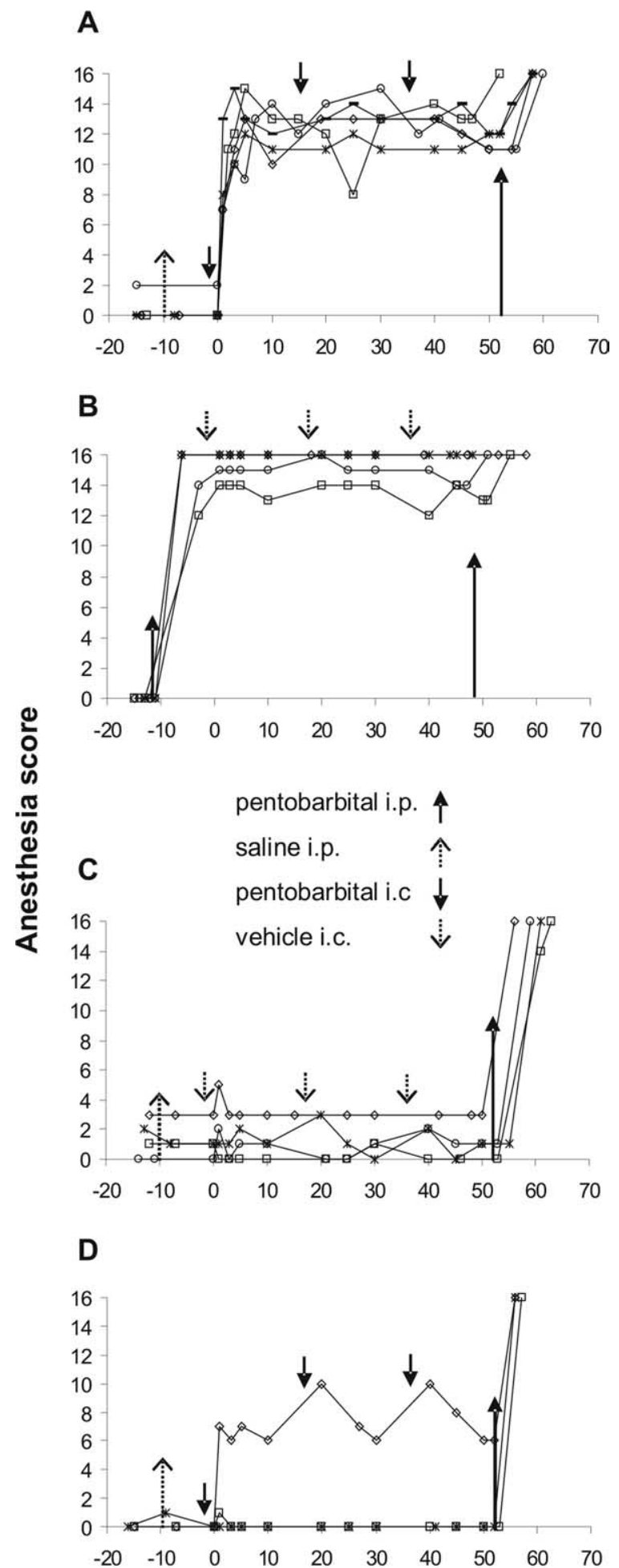

Minutes after first microinjection

Figure 2. Anesthesia score during the $50 \mathrm{~min}$ observation period in the four experimental groups in which pentobarbital or vehicle was microinjected into the MPTA. $A$, MPTA group. $B$, IP group. $C$, Awake group. D, Microinjection (-) group. Each line represents an individual animal. Upward-pointing arrows indicate intraperitoneal injections of either pentobarbital (solid arrows) or saline (dashed arrows) given before the observation period and of pentobarbital (long solid arrows) given at the end of the observation period as a prelude to perfusion. Downwardpointing arrows indicate intracerebral microinjection of pentobarbital (solid arrows) or vehicle (dashed arrows). The location of these arrows marks the average of the injection times of all animals in that group. Time 0 on the $x$-axis represents the time of completion of the first intracerebral (bilateral) microinjection. not differ significantly from that of the IP group in any of the four cortical areas quantified.

\section{Laminar differences in FOS-IR labeling}

FOS immunolabeling in the neocortex did not appear to be uniform across the cortical laminae. Focusing on SM1 cx, we marked all FOS-immunoreactive neurons from the pia to the subcortical white matter (Fig. 5A-C). FOS-immunoreactive nuclei were encountered at all depths, but very few were present in the superficial molecular layer (Fig. 5A, ROI 1). Density was somewhat higher, although not significantly so, in the supragranular (Fig. $5 A$, ROIs $2+3$ ) and subgranular (Fig. $5 A$, ROIs $5+6$ ) layers than at intermediate depths [that approximately corresponded to the granular layer 4 (Fig. 5A, ROI 4,D)]. This pattern was seen in all four experimental groups (16 rats), and is consistent with the observations of Lu et al. (2008) in awake animals.

\section{FOS expression in subcortical nuclei}

We scanned subcortical structures broadly for regions of differential FOS immunoreactivity in the MPTA, IP, and awake groups. We then made quantitative measurements on a subset of structures, with special attention to nuclei thought to be related to arousal state and to pain. Baseline immunolabeling in awake animals varied from region to region. In most nuclei sampled, however, there were no significant differences among the three groups (Fig. $4 B, C$ ). These included areas in which a priori we had anticipated that striking differences might emerge such as the ITh and MTh (which included the centrolateral, paracentral nuclei, paraventricular, intermediodorsal, and centromedial nuclei), the A5 noradrenergic cell group (identified by TH-IR double labeling) (Fig. 3E), the LC, and the GiA. We acknowledge, however, that given the limited number of animals per group we may have missed some regions that harbored real differences. Four subcortical areas were identified in which FOS-IR differed significantly among the MPTA, IP, and awake groups. These were the lateral habenular nucleus (LHb), the TMN, the ZI, and the Rpa. Interestingly, in two of these, results in the IP and the MPTA groups differed significantly.

\section{$L H b$}

This dorsomedial diencephalic nucleus is thought to be involved in nociceptive processing (Nagao et al., 1993; Matsumoto et al., 1994; Lehner et al., 2004) and other affective functions (Lee and Huang, 1988; Zhang et al., 2005). FOS expression was substantially increased during systemic pentobarbital anesthesia compared with the wake group $(p<0.01)$, consistent with earlier reports (Krukoff et al., 1992; Takayama et al., 1994; Ryabinin et al., 2000; Lu et al., 2008). However, no such increase occurred in the anesthesia-like state induced by intracerebral pentobarbital (MPTA group, $p>0.2$ compared with awake, $p<0.01$ compared with IP) (Figs. $3 C, 4 B)$. The microinjection $(-)$ group also showed no change compared with the MPTA group $(p>0.05)$ (Fig. 4D).

\section{TMN}

This histaminergic cell group in the caudal diencephalon is thought to promote arousal and to have a key role in the sedative response to GABAergic anesthetics (Lin et al., 1989, 1996; Nelson et al., 2002; Ko et al., 2003). Exposure of the TMN to $G_{A B A}-R$ active anesthetics (propofol, pentobarbital, muscimol) induces sedation (and LORR in the case of muscimol). Moreover exposure of the TMN to gabazine, a $\mathrm{GABA}_{\mathrm{A}}$-receptor antagonist, attenuates the sedation induced by systemically administered 
propofol and pentobarbital (Nelson et al., 2002). Like Nelson et al. (2002) and Lu et al. (2008), we found a high baseline level of FOS expression in the TMN (awake group), which was significantly reduced during systemic pentobarbital anesthesia (IP group, $p<0.001$ ). However, there was no reduction in FOS-IR during the anesthesia-like state induced by microinjection of pentobarbital in the MPTA group ( $p>0.2$ compared with awake, $p<$ 0.001 compared with IP) (Figs. 3D, $4 B$ ). No difference was found between the microinjection (-) group and the MPTA group $(p>0.2)$ (Fig. $4 D)$. A similar pattern of effects was noted for the LC and the GiA, but these differences were not significant.

\section{ZI}

This lateral diencephalic region is reciprocally connected to the MPTA (Sukhotinsky et al., 2007), and is thought to be involved in arousal, attention, posturelocomotion, and visceral functions (Mitrofanis, 2005). FOS expression in the awake group was low, and fell to near zero in both anesthetized groups. The decline was statistically significant in the IP group $(p<0.05)$, although not in the MPTA group $(p>0.05)$ (Fig. $4 C$ ).

\section{Rpa}

This serotonergic medullary nucleus is implicated in nociceptive and autonomic modulation (Mason, 2001; Nason and Mason, 2004). FOS-IR in the Rpa was relatively high in the awake state but was significantly reduced by systemic pentobarbital (IP group, $p<0.01$ ) (Fig. $4 C$ ). In the MPTA group, there was a reduction but it was not significant ( $p=0.2$ compared with both awake and IP groups). The difference in baseline FOS-IR and the effect of anesthesia between the Rpa and the GiA is noteworthy.

\section{Control observations}

In some experiments, microinjection of pentobarbital into the MPTA failed to induce a criterion level of anesthesia. This has been the experience in previous studies as well (Devor and Zalkind, 2001; Voss et al., 2005). The reasons are uncertain, but may relate to interanimal variability in the exact location of the relevant target neurons within the MPTA region or damage to some of these cells in previous microinjection trials. Such failed trials allowed us to evaluate whether changes in FOS expression are due to the microinjection procedure, including the drug deposited, or whether they are functionally related to action of the drug on behavior. We quantified FOS-IR in three such rats [microinjection (-) group]. Overall, FOS immunoreactivity resembled that seen in awake animals; in three of the four cortical ROIs it was significantly greater than in the microinjected rats in which an anesthesia-like state was successfully induced (i.e., the MPTA group). The exception was the SM1 cx, in which the difference did not reach statistical significance (Fig. 4D). Immunolabeling in the subcortical regions analyzed of the microinjection $(-)$ group did not differ significantly from the MPTA group. This observation is not surprising considering that there was also no significant difference between the MPTA and the awake groups in those areas (Fig. $4 B-D$ ).

\section{FOS-behavior correlations}

Mean anesthesia scores during the $50 \mathrm{~min}$ observation period differed most between the two anesthetized groups (MPTA and IP groups) and the two awake groups (awake and microinjection $(-)$ groups). However, there was variability among individual rats within all of the groups (Fig. 2), and this can provide additional information on the relation between FOS expression and arousal state. We plotted the density of FOS-immunoreactive neurons against total integrated anesthesia score for each individual animal, for all of the ROIs. This permitted us to determine 

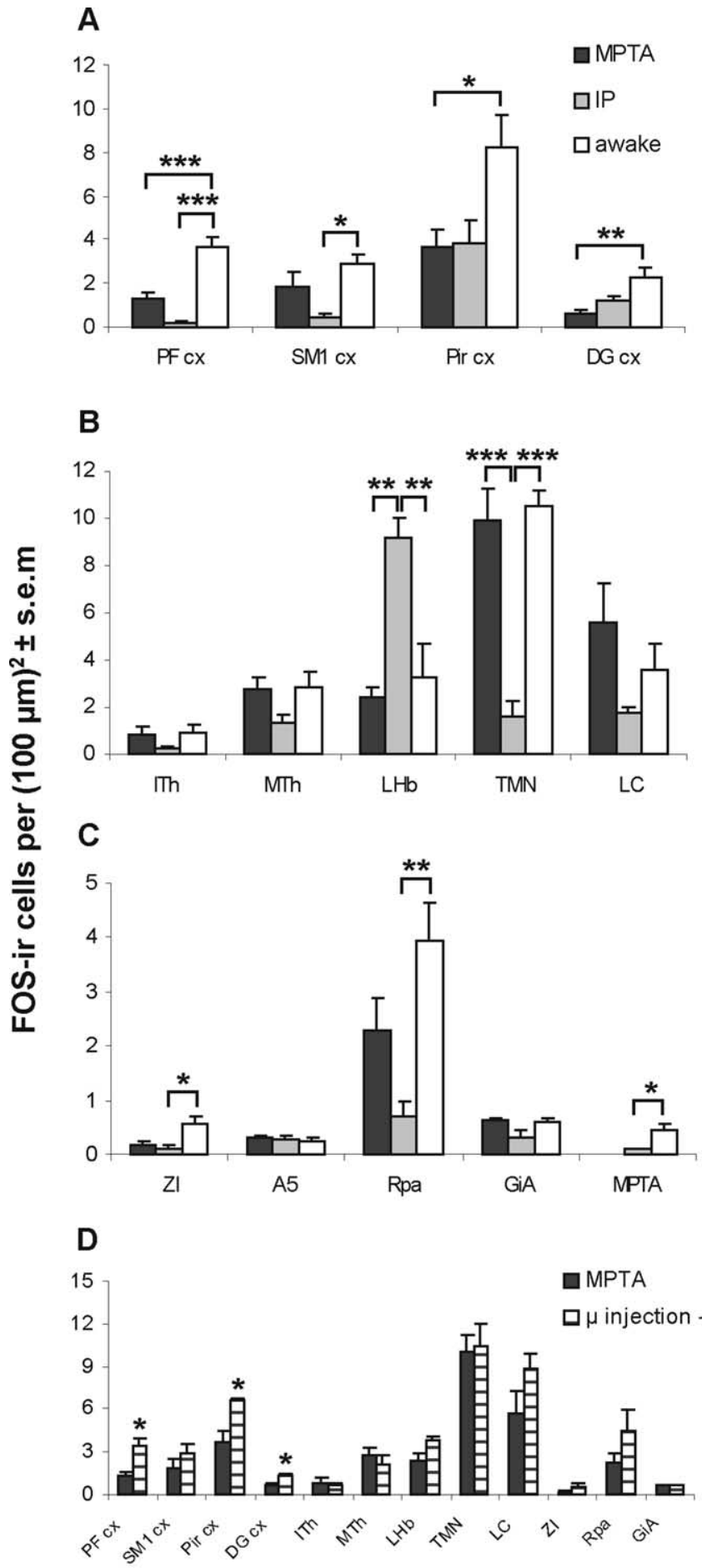

\section{Brain areas}

Figure 4. The mean density of FOS-immunoreactive neurons is affected by anesthesia in some, but not all, brain areas examined. $A-C$, Counts were made during the MPTA-induced anesthesia-like state (MPTA group), systemic pentobarbital anesthesia (IP group), and in the awake state (awake group). A, Cortical areas. B, C, Subcortical areas. Measurements in the MPTA were made in animals without intracerebral cannulae (see "supplemental groups" in the text). D. Comparison of FOS-IR density in animals in which microinjection of pentobarbital into the MPTA induced (MPTA group) or failed to induce [microinjection ( - ) group] an anesthesialike state. The number of rats sampled was as follows: IP and awake groups, $n=4$; microinjection ( - ) group, $n=3$; MPTA group, $n=4$ except for $n=5$ in the PF $c x$, SM1 cx, Pir cx, and Zl, and $n=3$ in the LC. ${ }^{*} p<0.05,{ }^{* *} p<0.01,{ }^{* * *} p<0.001$.
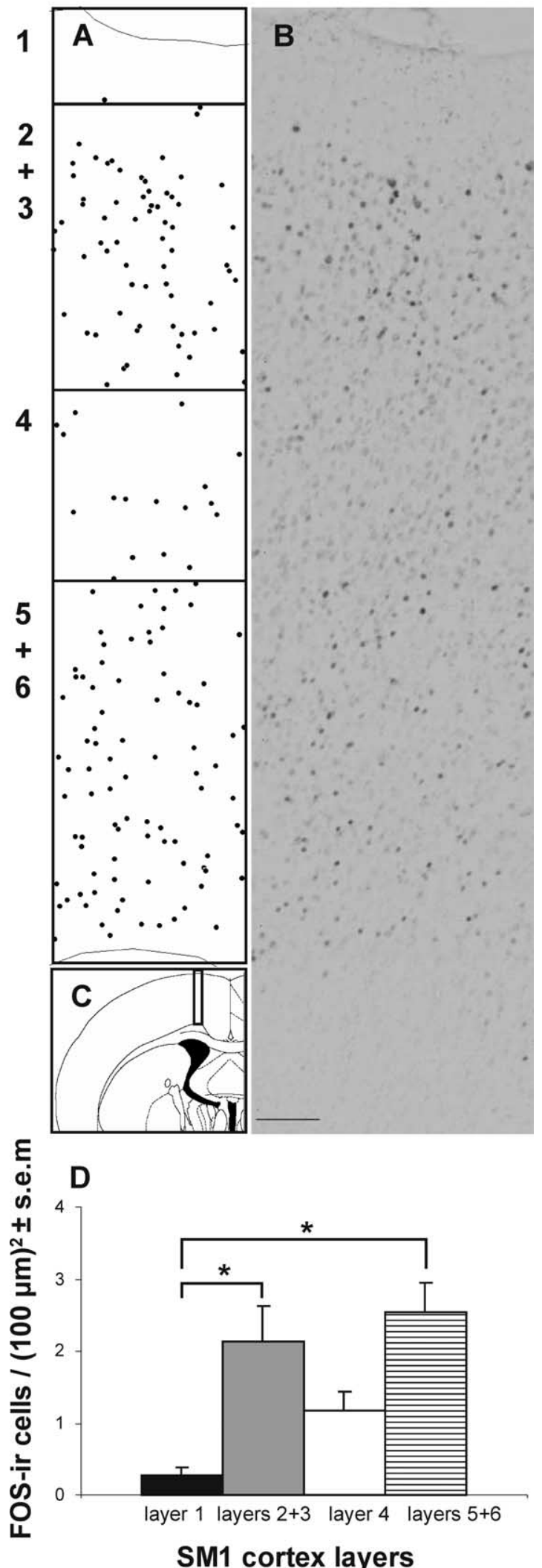

Figure 5. The density of FOS-immunoreactive neurons varies with laminar depth in the cortex. $A, B$, Plot and photomicrograph showing the distribution of immunolabeled neurons in SM1 $\mathrm{Cx}$ in an awake rat, ranging from the pial surface (top) to the subcortical white matter (bottom), $B=-0.92$. Each symbol in $A$ represents one FOS-immunoreactive cell. The rectangle in $\boldsymbol{C}$ shows the approximate location from which $\boldsymbol{A}$ and $\boldsymbol{B}$ were taken. In $\boldsymbol{D}$, values from each rat in all of the experimental groups are averaged. ${ }^{*} p<0.05$. Scale bar, $100 \mu \mathrm{m}$. 
whether there were regions with a particularly stringent dependency of behavior on neural activity. In all four cortical areas quantified we found a significant negative correlation between immunolabeling and depth of anesthesia (Fig. 6, Table 2). In the subcortical nuclei there was significant negative correlation for the ZI, the TMN, and the Rpa ( $p=0.005,0.004,0.0005$, respectively). In one nucleus, the $\mathrm{LHb}$, there was a significant positive correlation $(p<0.05)$. The ITh, MTh, LC, A5, and the GiA did not show a significant correlation ( $p>0.1)$. Overall, the most striking correlations occurred in the PF cx $(r=-0.94$, $\left.p=5.5 \times 10^{-8}\right)$, the Rpa $(r=-0.79, p=$ $0.0005)$, and the TMN $(r=-0.69, p=$ 0.004).

Correlations between FOS-IR and behavior were also calculated separately based on data from the motor (posture and LORR) and sensory (response to pinch) scales separately. For most ROIs correlation coefficients were quite similar for the two scores considered separately, or combined (Table 2 ). The only notable exceptions were in the DG cx, where neuronal activity correlated with motor suppression but not with antinociception (sensory), and the LHb, where correlation to antinociception was greater than to motor suppression.

\section{FOS-FOS correlations}

Another potential source of information is in joint correlations between ROIs. To this end, we ran a partial correlation coefficient analysis, which eliminates overall arousal state as a confounding variable. Here, for each brain region quantified, we calculated the vertical deviation of each data point (where data points are the measurement of FOS-IR in each animal) from the previously plotted linear regression line of FOS-IR on anesthesia score (Fig. 6). These values were then used to generate pairwise correlations between the regions. Results are shown in Figure 7. Overall there were 11 pairwise correlations with uncorrected $p<0.05$, and one correlation that remained significant after correction for multiple comparisons. This was the combination of SM1 cx and PF cx ( $p=5.37 \times 10^{-5}$ uncorrected, $p<0.005$ Bonferroni corrected).

\section{FOS expression in the MPTA}

The tissue bordering the defect caused by the implanted guide and injection cannulae, and the region of the microinjection itself, contained many FOS-immunoreactive neurons, including within the MPTA proper. Such labeling was also present in the pia crossed by the injection cannula (dorsal surface of the midbrain and pons). Since such labeling was present following microinjection of pentobarbital or vehicle, and whether or not anesthesia was induced, we attribute it to nonspecific cellular activation and/or trauma-evoked $\mathrm{Ca}^{2+}$ entry (Nozaki et al., 1992; Herrera and Robertson, 1996; Cirelli and Tononi, 2000). Interestingly, the presence of many such cells in both the MPTA and microinjec-

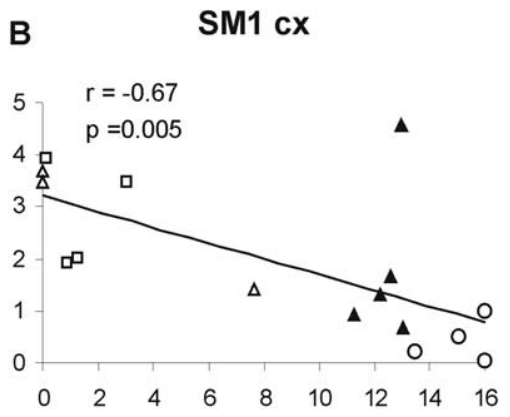

LHb

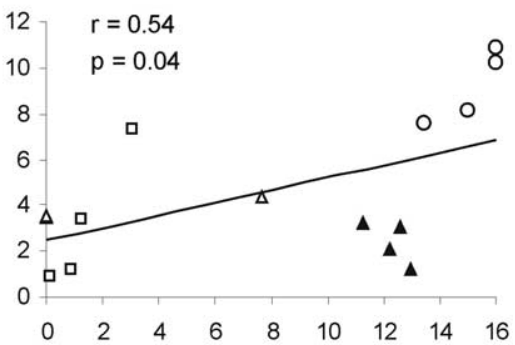

$\mathrm{F}$

$\mathrm{RPa}$

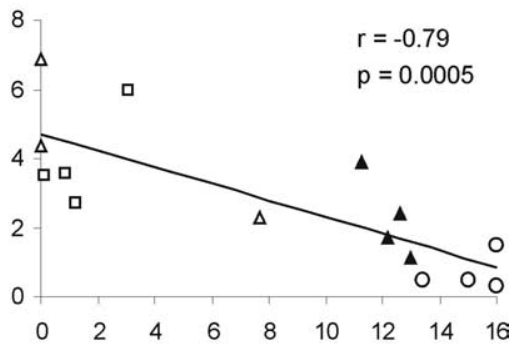

Integrated anesthesia score

Figure 6. In some brain areas there was a significant correlation between anesthesia score (integrated over the 50 min observation period) and the density of FOS-immunoreactive neurons. Symbols represent values of individual rats in the four $p$ value. See Table 2 for additional ROls.

tion $(-)$ groups indicates that high concentrations of pentobarbital do not necessarily preclude FOS expression under these circumstances. Because of the local tissue damage present, we were not able to use these sections to quantitatively evaluate effects of pentobarbital on intact neurons in the MPTA.

FOS expression was therefore examined in two supplemental groups that did not have implanted guide cannulae. These received an anesthetic dose of pentobarbital intraperitoneally or an equivalent volume of saline, and remained awake. Relatively low levels of FOS expression were seen in the awake animals $[0.45 \pm$ 0.1 SEM per $\left.(100 \mu \mathrm{m})^{2}\right]$, but even fewer occurred during systemic pentobarbital anesthesia $\left[0.11 \pm 0.01\right.$ SEM per $(100 \mu \mathrm{m})^{2}$; $p<0.05$ ] (Figs. 4C, 8).

\section{FOS expression in the ventricular ependyma}

An incidental observation was intense FOS immunolabeling of ependymal cell nuclei in the interventricular aqueduct and the fourth ventricle in some of the rats (Fig. 9). This pattern of labeling was seen in six of eight animals microinjected with pentobarbital, regardless of whether an anesthesia-like state was generated. 
Table 2. Coefficient of correlation between anesthesia score (total, and separately for motor and sensory scales; integrated over the 50 min observation period) and F0S-ir cell density in all ROIs, based on data from all experimental groups $[n=16$ rats, MPTA $=5, I P=4$, awake $=4$, microinjection $(-)=3]$

\begin{tabular}{|c|c|c|c|c|c|c|}
\hline \multirow[b]{2}{*}{$\mathrm{ROI}$} & \multicolumn{2}{|l|}{ Total score } & \multicolumn{2}{|l|}{ Motor } & \multicolumn{2}{|l|}{ Sensory } \\
\hline & Correlation coefficient ( $r$ ) & $p$ value & Correlation coefficient $(r)$ & $p$ value & Correlation coefficient $(r)$ & $p$ value \\
\hline$P F C X$ & -0.94 & $5.5 \times 10^{-8}$ & -0.92 & $3.98 \times 10^{-7}$ & -0.94 & $1.15 \times 10^{-7}$ \\
\hline Rpa & -0.79 & 0.0005 & -0.79 & 0.0004 & -0.76 & 0.001 \\
\hline TMN & -0.69 & 0.004 & -0.65 & 0.008 & -0.71 & 0.003 \\
\hline $\mathrm{Zl}$ & -0.67 & 0.005 & -0.62 & 0.011 & -0.7 & 0.003 \\
\hline SM1 Cx & -0.67 & 0.005 & -0.66 & 0.005 & -0.65 & 0.006 \\
\hline Pir cx & -0.66 & 0.005 & -0.69 & 0.003 & -0.61 & 0.012 \\
\hline $\mathrm{LHb}$ & 0.54 & 0.037 & 0.46 & 0.087 & 0.62 & 0.014 \\
\hline$D G C x$ & -0.52 & 0.046 & -0.61 & 0.016 & -0.41 & 0.13 \\
\hline $\mathrm{GiA}$ & -0.42 & 0.12 & -0.39 & 0.15 & -0.44 & 0.10 \\
\hline $\mathrm{LC}$ & -0.38 & 0.18 & -0.31 & 0.28 & -0.45 & 0.11 \\
\hline A5 & 0.38 & 0.22 & 0.34 & 0.28 & 0.43 & 0.17 \\
\hline MTh & -0.25 & 0.38 & -0.29 & 0.29 & -0.19 & 0.50 \\
\hline ITh & -0.22 & 0.44 & -0.27 & 0.33 & -0.15 & 0.59 \\
\hline
\end{tabular}

Significant correlations $(p<0.05)$ are indicated in bold.

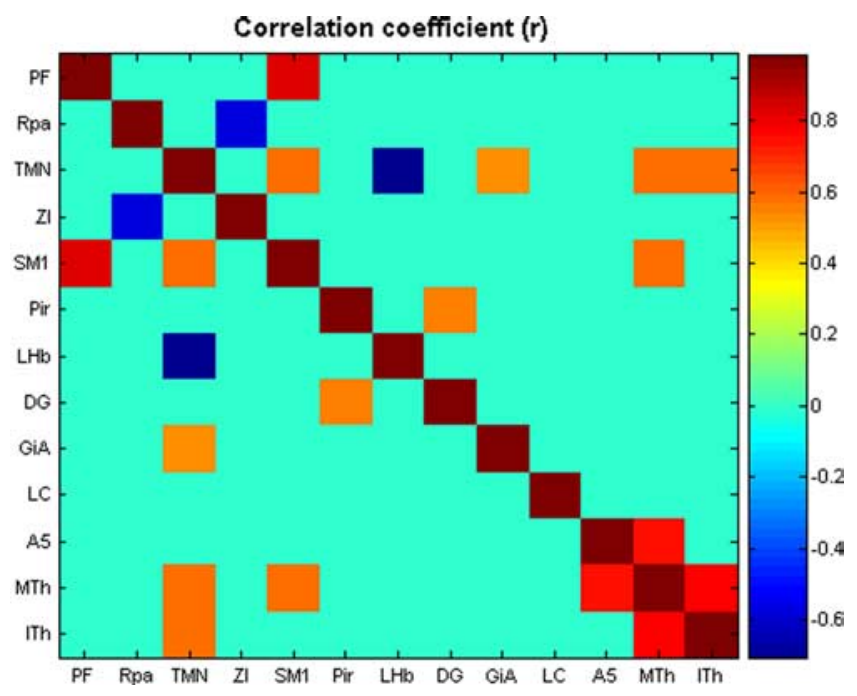

Figure 7. Partial correlation between pairs of ROIs from density of FOS-IR. Correlation coefficients are coded as indicated in the panel on the right. All values of $r$ that yielded $p>0.05$ were colored according to $r=0$.

Labeling did not occur in one of the MPTA group animals, and in one microinjection ( - ) group animal that obtained a very low anesthesia score. In contrast, only one of the eight animals microinjected with vehicle (the awake and IP groups) showed FOS-IR in the ependyma, even when anesthesia was present (the exceptional animal was in the awake group; Fisher test, $p<0.05$ ). In most of the animals with immunolabeling in the aqueduct and fourth ventricle, FOS-immunoreactive ependymal cells also appeared in the third ventricle, and in some also in the region of the foramen of Monroe and in the adjacent parts of the lateral ventricle (four of seven rats). Labeling density within the lateral ventricle appeared to decline with distance from the foramen.

In the animals with ependymal labeling, there were also FOSimmunoreactive nuclei in the pia surrounding the entire brainstem, and not restricted to its dorsal surface. This labeling did not extend to the pia over the cerebellar or cerebral cortices. Endothelial cells lining intracerebral blood vessels were not labeled. This pattern of immunoreactivity suggests that pentobarbital microinjection led to the release or generation of a diffusible substance in the cerebral spinal fluid capable of inducing $c$-fos expression in ependymal and pial cells.

\section{The kinetics of FOS quenching}

$c$-fos is an immediate early gene. Transient neural activation increases $c$-fos mRNA levels after a few minutes with expression peaking at 15-30 min (Sheng and Greenberg, 1990; Hoffman et al., 1993; Chaudhuri, 1997; Tononi and Cirelli, 2001). FOS, the protein product of $c$-fos expression, is generally detectable within $\sim 30 \mathrm{~min}$, and it persists for several hours (Dragunow and Faull, 1989; Hoffman et al., 1993). It is less clear, however, how rapidly baseline FOS levels dissipate when neural activity is suppressed. Some information is available from the animals in the awake group. These animals were exposed to pentobarbital from the end of the observation period until tissue fixation, an interval that lasted $11,11,12$, and $13 \mathrm{~min}$ (minus the 1-2 min required for anesthesia induction). To evaluate whether the level of FOS protein might already have begun to decline during this time, we anesthetized an additional five rats (without cannula assemblies) that had been awake for at least $50 \mathrm{~min}$. In these rats we allowed 8 , $8,11,13$, and $13 \mathrm{~min}$ to pass from the moment of pentobarbital injection until fixative reached the brain. No significant change in FOS expression was noted over this 5 min interval $(p>0.2$, measurements made in the supragranular SM1 cx and in the MPTA). It is likely, therefore, that visible decline from the wake state begins after a latency of $>13$ min (see Discussion).

\section{Discussion}

Anesthetic agents delivered systemically distribute widely in the cerebral vasculature. It is generally presumed that the resulting anesthesia is a consequence of widespread suppression of neuronal activity due to direct binding of the agents to local membrane receptors. The discovery that an anesthesia-like state can be induced rapidly, reversibly, and in a $\mathrm{GABA}_{\mathrm{A}}$ - $\mathrm{R}$-dependent manner (Devor and Zalkind, 2001; Sukhotinsky et al., 2007) by local administration of pentobarbital and other $\mathrm{GABA}_{\mathrm{A}}-\mathrm{R}$-active agents in the MPTA suggests the alternative, that anesthetic drugs might act on a circumscribed population of neurons, which then trigger the functional components of anesthesia via specific neural pathways.

Despite the radically different drug distributions by systemic versus MPTA injection, behavioral effects were similar, and they yielded similar, widespread suppression of cortical activity with a robust correlation between FOS-IR and behavior. On the other hand, the change in FOS expression differed in some subcortical nuclei, with no obvious change observed in many others. This includes nuclei thought to be closely related to the control of vigilance in the context of sleep-wake transitions. 


\section{Patterns of FOS expression}

FOS is expressed rapidly following adequate stimulation of susceptible neuronal populations and is hence widely used as a marker of neuronal activity (Sheng and Greenberg, 1990; Hoffman et al., 1993). Less is known about the kinetics of FOS quenching when neuronal activity is suppressed. We found substantial quenching within $\sim 60 \mathrm{~min}$ of anesthesia $(50 \mathrm{~min}$ observation period plus the time required for tissue fixation by perfusion). The absence of a detectable difference between awake rats perfused 8 versus 13 min after terminal anesthesia indicates that FOS expression had not yet begun to decline within this time frame. Thus, results from the awake group probably faithfully represent the status in animals actually awake.

Although we quantified FOS-IR quenching during anesthesia in only four cortical regions, qualitatively this change occurred throughout the cerebral mantle. But, changes were not uniform throughout. The correlation between FOS-IR and integrated anesthesia score was particularly striking in the PF cx, implying special salience of prefrontal regions for anesthesia induced unconsciousness. A significant overall correlation also occurred in the SM1 cx, although the FOS-IR reduction was not statistically significant in the MPTA group. This might reflect differences in the engagement of primary versus higher order cortical areas in the two modes of anesthesia. We note, however, that the joint partial correlation between PF cx and SM1 cx was particularly high, suggesting that these two areas act cooperatively. FOS-IR suppression was somewhat more complete overall during systemic than MPTA-induced anesthesia, perhaps because the depth of anesthesia in the MPTA group was slightly less (Fig. 2A,B). The observation of similar cortical suppression, despite very different drug distribution, is by no means a trivial or obvious result. It suggests that suppressed cortical activity is a potentially important common denominator in anesthesia and the anesthesia-like state induced by MPTA microinjection.

Subcortical and spinal structures also contribute to anesthesia, either directly or by secondary ascending effects. Correlation was found between FOS expression and state of alertness in four subcortical nuclei: LHb, TMN, ZI, and Rpa. But unlike the cerebral cortex, there were significant differences in FOS expression in the IP versus the MPTA groups in two of them ( $\mathrm{LHb}$ and TMN), and, in all, changes following MPTA microinjection were the same whether or not an anesthesia-like state was induced (Fig. $4 D)$. The LHb stood out as the only location identified in which FOS-IR significantly increased during systemic anesthesia. However, its activity did not change during MPTA-induced anesthesia. In the TMN there was considerable suppression of activity in the IP group (compared with awake), confirming Nelson et al. (2002) and Lu et al. (2008), but no change occurred in the MPTA group. FOS-IR is also not reduced in the TMN during ketamineinduced anesthesia (Lu et al., 2008). These dissociations undermine the conclusion that activity in the LHb and the TMN play an indispensable role in the loss of consciousness associated with anesthesia, at least for MPTA-induced anesthesia.

The medullary Rpa showed higher baseline FOS-IR than other component nuclei of the ventromedial medulla, and the ZI was also relatively active during wake. Like the cerebral cortex but unlike the LHb and TMN, activity in both nuclei was suppressed in the MPTA and the IP groups, although the reduction was statistically significant only in the IP group. The serotonergic brainstem raphe nuclei have been implicated in the ascending control of cerebral arousal, notably the dorsal raphe nucleus (DRN), as well as in descending control of spinal motor functions and nociception. Unfortunately, we were unable to evaluate FOS-IR in the DRN because of its proximity to the tissue defect caused by insertion of the injection cannulae.

We were surprised by the failure to observe a significant change in FOS-IR in the LC, A5, GiA, MTh, and ITh. Activity in these nuclei is clearly implicated in sleep-wake transitions (Saper et al., 2001; Tononi and Cirelli, 2001), with the nonspecific thalamic nuclei (MTh and ITh) constituting the best known portal for cortical arousal (McCormick and Bal, 1997; Novak and Nunez, 1998; Cirelli and Tononi, 2000; Alkire and Miller, 2005; Fiset et al., 2005). A natural inference is that some nuclei might be involved in loss of consciousness during sleep, but not during anesthesia and anesthesia-like states. This conclusion must remain tentative, however, given the relatively short duration of anesthesia in our experiments and the limited nature of our survey of subcortical sleep-related nuclei. For example, we did not 
examine the DRN, the mesopontine or basal forebrain cholinergic cell groups, or the orexin-expressing neurons of the tuberal hypothalamus. The ventrolateral preoptic nucleus (VLPO) has emerged as a master sleep-active nucleus capable of inhibiting a variety of subcortical arousal nuclei (Sherin et al., 1998; Chou et al., 2002; Gaus et al., 2002). It has been reported that FOS-IR in GABA and galanin-expressing VLPO neurons increases during anesthesia induced by systemic $\mathrm{GABA}_{\mathrm{A}}$ - $\mathrm{R}$-active agents [but not ketamine (Nelson et al., 2002; Lu et al., 2008)]. We examined the VLPO and found the same level of FOS-IR in the awake and the IP groups (mean density [labeled cells per $(100 \mu \mathrm{m})^{2}$ ] for awake group $=1.67 \pm 0.73 ;$ IP group $=1.64 \pm 0.67$ ) and only slightly more in the MPTA group $(3.24 \pm 0.87, p>0.05)$. But since we did not identify the GABA/galanin-expressing cells specifically, an increase in this population may have been obscured. Other possible reasons for the discrepancy are the shorter duration of anesthesia in our study as mention above, different time in the circadian cycle, and different pentobarbital dose.

\section{Cerebral activity, anesthesia, and anesthesia-like states}

In some areas, the two modes of drug delivery induced similar changes in neural activity compared with wake, while in others there were substantial differences. Similar response might indicate that the areas in question (cortex, ZI, and Rpa) are closely associated with anesthesia. Areas that respond differentially, the $\mathrm{LHb}$ and the TMN, might be not essential. Alternatively, they may be associated with the subtle differences in the anesthetic states induced by the two modes of drug administration. Such differences include the duration and the depth of anesthesia for example, or functions not quantified here such as memory inhibition and autonomic variables. The many areas in which no change in activity was observed might also be unrelated to anesthesia, or more likely, activity in these areas might simply not be adequately tracked by our FOS protocol.

The two modes of anesthesia yielded similar behavioral effects whereas changes in brain activity were similar in some areas and different in others. Two possible explanations arise. One is that the different patterns might have resulted from totally independent mechanisms: direct drug action in the IP group and ascending axonal pathways in the MPTA group (Sukhotinsky et al., 2007). On the other hand, it is possible that the same mechanism is involved in both modes of anesthesia. Specifically, systemically administered barbiturates might not act exclusively by binding to receptors on cortical neurons but rather, at least in part, by a primary action on MPTA neurons. Cortical suppression would then occur secondarily, as in the MPTA group, via ascending pathways. A precedent for this is analgesia induced by systemic opiates. Circulating opiates act on cells in the midbrain periaqueductal gray and effect analgesia via (descending) axonal pathways (Basbaum and Fields, 1984). The possibility of a primary action of circulating barbiturates in the MPTA is consistent with the observation that FOS-IR in the MPTA is dramatically suppressed during systemic-induced general anesthesia. In the pathway model, the primary (MPTA) and secondary (pathway) events are expected to occur in rapid succession, making them difficult to dissociate in time. Unfortunately, since FOS quenching requires a long period of integration, this method cannot be used to determine whether MPTA suppression happens first, followed later by cortical suppression. Functional imaging studies in volunteers anesthetized with systemic agents have the same limitation. Methods with better temporal resolution will be required to determine the sequence of events. It should be noted, however, that imaging in humans routinely reveals altered activity in an upper mesopontine region consistent with the MPTA (Paus, 2000; Parvizi and Damasio, 2003).

\section{References}

Alkire MT, Miller J (2005) General anesthesia and the neural correlates of consciousness. Prog Brain Res 150:229-244.

Alkire MT, Haier RJ, Fallon JH (2000) Toward a unified theory of narcosis: brain imaging evidence for a thalamocortical switch as the neurophysiologic basis of anesthetic-induced unconsciousness. Conscious Cogn 9:370-386.

Antkowiak B (1999) Different actions of general anesthetics on the firing patterns of neocortical neurons mediated by the GABA(A) receptor. Anesthesiology 91:500-511.

Basbaum AI, Fields HL (1984) Endogenous pain control systems: brainstem spinal pathways and endorphin circuitry. Annu Rev Neurosci 7:309-338.

Chaudhuri A (1997) Neural activity mapping with inducible transcription factors. Neuroreport 8:iii-vii.

Chou TC, Bjorkum AA, Gaus SE, Lu J, Scammell TE, Saper CB (2002) Afferents to the ventrolateral preoptic nucleus. J Neurosci 22:977-990.

Cirelli C, Tononi G (2000) On the functional significance of c-fos induction during the sleep-waking cycle. Sleep 23:453-469.

Devor M, Zalkind V (2001) Reversible analgesia, atonia, and loss of consciousness on bilateral intracerebral microinjection of pentobarbital. Pain 94:101-112.

Dragunow M, Faull R (1989) The use of c-fos as a metabolic marker in neuronal pathway tracing. J Neurosci Methods 29:261-265.

Fiset P, Plourde G, Backman SB (2005) Brain imaging in research on anesthetic mechanisms: studies with propofol. Prog Brain Res 150:245-250.

Franks NP (2008) General anaesthesia: from molecular targets to neuronal pathways of sleep and arousal. Nat Rev Neurosci 9:370-386.

Franks NP, Lieb WR (1994) Molecular and cellular mechanisms of general anaesthesia. Nature 367:607-614.

Gaus SE, Strecker RE, Tate BA, Parker RA, Saper CB (2002) Ventrolateral preoptic nucleus contains sleep-active, galaninergic neurons in multiple mammalian species. Neuroscience 115:285-294.

Haycock JW (1987) Stimulation-dependent phosphorylation of tyrosine hydroxylase in rat corpus striatum. Brain Res Bull 19:619-622.

Hayes RL, Pechura CM, Katayama Y, Povlishock JT, Giebel ML, Becker DP (1984) Activation of pontine cholinergic sites implicated in unconsciousness following cerebral concussion in the cat. Science 223:301-303.

Hentschke H, Schwarz C, Antkowiak B (2005) Neocortex is the major target of sedative concentrations of volatile anaesthetics: strong depression of firing rates and increase of GABAA receptor-mediated inhibition. Eur J Neurosci 21:93-102.

Herrera DG, Robertson HA (1996) Activation of c-fos in the brain. Prog Neurobiol 50:83-107.

Hoffman GE, Smith MS, Verbalis JG (1993) c-Fos and related immediate early gene products as markers of activity in neuroendocrine systems. Front Neuroendocrinol 14:173-213.

Hubel DH, Wiesel TN (1959) Receptive fields of single neurones in the cat's striate cortex. J Physiol 148:574-591.

Katayama Y, Young HF, Dunbar JG, Hayes RL (1988) Coma associated with flaccidity produced by fluid-percussion concussion in the cat. II: Contribution of activity in the pontine inhibitory system. Brain Inj 2:51-66.

Ko EM, Estabrooke IV, McCarthy M, Scammell TE (2003) Wake-related activity of tuberomammillary neurons in rats. Brain Res 992:220-226.

Koningsmark BW (1970) Methods for the counting of neurons. In: Contemporary research methods in neuroanatomy (Nauta WJH, Ebbesson SOE, eds), pp 315-340. New York: Springer.

Krukoff TL, Morton TL, Harris KH, Jhamandas JH (1992) Expression of $\mathrm{c}$-fos protein in rat brain elicited by electrical stimulation of the pontine parabrachial nucleus. J Neurosci 12:3582-3590.

Lee EH, Huang SL (1988) Role of lateral habenula in the regulation of exploratory behavior and its relationship to stress in rats. Behav Brain Res 30:265-271.

Lehner M, Taracha E, Skórzewska A, Wisłowska A, Zienowicz M, Maciejak P, Szyndler J, Bidziński A, Płaźnik A (2004) Sensitivity to pain and c-Fos expression in brain structures in rats. Neurosci Lett 370:74-79.

Lin JS, Sakai K, Vanni-Mercier G, Jouvet M (1989) A critical role of the posterior hypothalamus in the mechanisms of wakefulness determined by microinjection of muscimol in freely moving cats. Brain Res 479:225-240. Lin JS, Hou Y, Sakai K, Jouvet M (1996) Histaminergic descending inputs to 
the mesopontine tegmentum and their role in the control of cortical activation and wakefulness in the cat. J Neurosci 16:1523-1537.

Lu J, Nelson LE, Franks N, Maze M, Chamberlin NL, Saper CB (2008) Role of endogenous sleep-wake and analgesic systems in anesthesia. J Comp Neurol 508:648-662.

Lydic R, Baghdoyan HA (2005) Sleep, anesthesiology, and the neurobiology of arousal state control. Anesthesiology 103:1268-1295.

Magni F, Moruzzi G, Rossi G, Zanchetti A (1959) EEG arousal following inactivation of the lower brainstem by selective injection of barbiturate into the vertebral circulation. Arch Ital Biol 97:33-46.

Mason P (2001) Contributions of the medullary raphe and ventromedial reticular region to pain modulation and other homeostatic functions. Annu Rev Neurosci 24:737-777.

Matsumoto N, Yahata F, Kawarada K, Kamata K, Suzuki TA (1994) Tooth pulp stimulation induces c-fos expression in the lateral habenular nucleus of the cat. Neuroreport 5:2397-2400.

McCormick DA, Bal T (1997) Sleep and arousal: thalamocortical mechanisms. Annu Rev Neurosci 20:185-215.

Mitrofanis J (2005) Some certainty for the "zone of uncertainty"? Exploring the function of the zona incerta. Neuroscience 130:1-15.

Morales FR, Sampogna S, Rampon C, Luppi PH, Chase MH (2006) Brainstem glycinergic neurons and their activation during active (rapid eye movement) sleep in the cat. Neuroscience 142:37-47.

Nagao M, Kamo H, Akiguchi I, Kimura J (1993) Induction of c-Fos-like protein in the lateral habenular nucleus by persistent noxious peripheral stimulation. Neurosci Lett 151:37-40.

Nason MW Jr, Mason P (2004) Modulation of sympathetic and somatomotor function by the ventromedial medulla. J Neurophysiol 92:510-522.

Nelson LE, Guo TZ, Lu J, Saper CB, Franks NP, Maze M (2002) The sedative component of anesthesia is mediated by GABA(A) receptors in an endogenous sleep pathway. Nat Neurosci 5:979-984.

Novak CM, Nunez AA (1998) Daily rhythms in Fos activity in the rat ventrolateral preoptic area and midline thalamic nuclei. Am J Physiol 275:R1620-R1626.

Nozaki K, Boccalini P, Moskowitz MA (1992) Expression of c-fos-like immunoreactivity in brainstem after meningeal irritation by blood in the subarachnoid space. Neuroscience 49:669-680.

Ommaya AK, Gennarelli TA (1974) Cerebral concussion and traumatic unconsciousness. Correlation of experimental and clinical observations of blunt head injuries. Brain 97:633-654.

Parvizi J, Damasio AR (2003) Neuroanatomical correlates of brainstem coma. Brain 126:1524-1536.

Paus T (2000) Functional anatomy of arousal and attention systems in the human brain. Prog Brain Res 126:65-77.

Paxinos G, Watson C (1986) The rat brain in stereotaxic coordinates, Ed 2. San Diego: Academic.

Paxinos G, Watson C (1998) The rat brain in stereotaxic coordinates, Ed 4. San Diego: Academic.
Plourde G, Belin P, Chartrand D, Fiset P, Backman SB, Xie G, Zatorre RJ (2006) Cortical processing of complex auditory stimuli during alterations of consciousness with the general anesthetic propofol. Anesthesiology 104:448-457.

Plum F, Posner JB (1980) The diagnosis of stupor and coma. Philadelphia: Davis.

Pompeiano M, Cirelli C, Tononi G (1994) Immediate-early genes in spontaneous wakefulness and sleep: expression of c-fos and NGFI-A mRNA and protein. J Sleep Res 3:80-96.

Ryabinin AE, Wang YM, Bachtell RK, Kinney AE, Grubb MC, Mark GP (2000) Cocaine- and alcohol-mediated expression of inducible transcription factors is blocked by pentobarbital anesthesia. Brain Res 877:251-261.

Saper CB, Chou TC, Scammell TE (2001) The sleep switch: hypothalamic control of sleep and wakefulness. Trends Neurosci 24:726-731.

Sheng M, Greenberg ME (1990) The regulation and function of c-fos and other immediate early genes in the nervous system. Neuron 4:477-485.

Sherin JE, Elmquist JK, Torrealba F, Saper CB (1998) Innervation of histaminergic tuberomammillary neurons by GABAergic and galaninergic neurons in the ventrolateral preoptic nucleus of the rat. J Neurosci 18:4705-4721.

Sukhotinsky I, Zalkind V, Lu J, Hopkins DA, Saper CB, Devor M (2007) Neural pathways associated with loss of consciousness caused by intracerebral microinjection of GABA A-active anesthetics. Eur J Neurosci 25:1417-1436.

Surtees R, Leonard JV (1989) Acute metabolic encephalopathy: a review of causes, mechanisms and treatment. J Inherit Metab Dis 12 [Suppl 1]:42-54.

Takayama K, Suzuki T, Miura M (1994) The comparison of effects of various anesthetics on expression of Fos protein in the rat brain. Neurosci Lett 176:59-62.

Tononi G, Cirelli C (2001) Modulation of brain gene expression during sleep and wakefulness: a review of recent findings. Neuropsychopharmacology 25:S28-35.

Tung A, Mendelson WB (2004) Anesthesia and sleep. Sleep Med Rev 8:213-225.

Voss LJ, Young BJ, Barnards JP, Sleigh J (2005) Differential anaesthetic effects following microinjection of thiopentone and propofol into the pons of adult rats: a pilot study. Anaesth Intensive Care 33:373-380.

Zecharia AY, Nelson LE, Gent TC, Schumacher M, Jurd R, Rudolph U, Brickley SG, Maze M, Franks NP (2009) The involvement of hypothalamic sleep pathways in general anesthesia: testing the hypothesis using the GABAA receptor beta3N265M knock-in mouse. J Neurosci 29:2177-2187.

Zhang F, Zhou W, Liu H, Zhu H, Tang S, Lai M, Yang G (2005) Increased c-Fos expression in the medial part of the lateral habenula during cueevoked heroin-seeking in rats. Neurosci Lett 386:133-137.

Zimmermann M (1983) Ethical guidelines for investigations of experimental pain in conscious animals. Pain 16:109-110. 